\title{
Author Correction: A genome-wide cross-trait analysis from UK Biobank highlights the shared genetic architecture of asthma and allergic diseases
}

\author{
Zhaozhong Zhu, Phil H. Lee, Mark D. Chaffin, Wonil Chung (D), Po-Ru Loh (D), Quan Lu, David C. Christiani and Liming Liang (D)
}

Correction to: Nature Genetics https://doi.org/10.1038/s41588-018-0121-0, published online 21 May 2018.

In the version of this article originally published, there were two errors in the text of the second paragraph of the Methods section. In the sentence "To identify genetic variants that contribute to doctor-diagnosed asthma and allergic diseases (detailed phenotype information described in the Supplementary Note) and link them with other conditions, we performed GWASs using phenotype measures in UK Biobank participants $(N=487,409)$ " the number of participants should have been 150,509 . In the sentence "Thus, a total of 110,361 European descendants with high-quality genotyping and complete phenotype/covariate data were used for these analyses, including 25,685 allergic diseases subjects (hay fever/allergic rhinitis or eczema, without doctor-diagnosed asthma), 14,085 asthma subjects and 76,768 controls for the analysis" the phrase "without doctor-diagnosed asthma" should have read "some with doctor-diagnosed asthma." The errors have been corrected in the HTML and PDF versions of the article.

Published online: 2 November 2018

https://doi.org/10.1038/s41588-018-0284-8

\section{Author Correction: Public resources aid diabetes gene discovery}

\author{
Diana L. Cousminer and Struan F. A. Grant
}

Correction to: Nature Genetics https://doi.org/10.1038/s41588-018-0242-5, published online 8 October 2018.

In the version of this article originally published, the text was incorrect in the first paragraph of the 'Remaining challenges' section. The first two sentences appeared as "Even though this current study substantially increases the number of loci associated with T2D, only approximately $18 \%$ of the genetic component of T2D risk is explained by the total complement of genetic variants uncovered genome wide in Mahajan et al. ${ }^{6}$. Interestingly, only a small proportion of that heritability was explained by low-frequency or rare variants $(1.1 \%)$, thus potentially indicating that many more of these variants still remain to be characterized in even larger sample sizes." However, they should have read "Even though this current study substantially increases the number of loci associated with T2D, only a proportion of the genetic component of T2D risk is explained by the total complement of genetic variants uncovered genome wide in Mahajan et al. ${ }^{6}$. Interestingly, only a relatively small proportion of that heritability was explained by low-frequency or rare variants, thus potentially indicating that many more of these variants still remain to be characterized in even larger sample sizes." The text has been corrected in the HTML and PDF versions of the paper.

Published online: 5 November 2018

https://doi.org/10.1038/s41588-018-0292-8

\section{Author Correction: Distinguishing genetic correlation from causation across 52 diseases and complex traits}

\author{
Luke J. O'Connor (D) and Alkes L. Price (D) \\ Correction to: Nature Genetics https://doi.org/10.1038/s41588-018-0255-0, published online 29 October 2018.
}

In the version of this article originally published, there were errors in equations. In the HTML and PDF, the initial term of equation 10 was estimated GCP but should have been estimated standard error, while a 'hat' was missing from the first alpha in the second term of the expression at the end of the paragraph following equation (6) in the Methods. In addition, in the abstract in the PDF, a subscript 1 was used instead of a subscript 2 for the final term of the first fourth-moment expression. These errors have been corrected in the HTML, $\mathrm{PDF}$ and print versions of the paper. 